year category, where there was no difference. Rates were higher amongst men in each of the three age categories $\geq 75$ years. Our analysis indicates that the disparity between hospitalisations for older men and women has narrowed over the period of our study, owing to increased hospitalisations in women and decreased hospitalisation in men.

Conclusion Our analysis of hospitalisation data has seen a convergence in rates between the sexes and a worrying rise in hospitalisations amongst women, particularly among those less than 70 years. This mirrors trends internationally where women have higher rates of COPD than men throughout most of their lifespan, although it appears that they are especially vulnerable before the age of 65 . It is known that women are more susceptible than men to lung damage from cigarette smoke and other pollutants, and recent evidence highlights that they have more severe COPD exacerbations than males, resulting in higher hospitalisations. This study confirms these trends and points to the urgent need to raise awareness of this preventable disease, and tackle the high smoking rates that still prevail in Ireland, particularly in socioeconomically deprived areas.

\section{P23 USING NATIONAL PHARMACY CLAIMS DATA TO ESTIMATE THE PREVALENCE AND PATTERNS OF MEDICATION USE FOR COPD IN IRELAND}

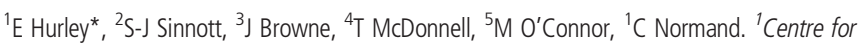
Health Policy and Management, Trinity College Dublin, Dublin, Ireland; ${ }^{2}$ Department of Non-communicable Disease Epidemiology, London School of Hygiene and Tropical Medicine, London, UK; ${ }^{3}$ School of Public Health, University College Cork, Cork, Ireland; ${ }^{4}$ School of Medicine, University College Dublin, Dublin, Ireland; ${ }^{5}$ Department of Public Health, Health Services Executive, Dublin, Ireland

\subsection{6/jech-2019-SSMabstracts. 174}

Background Ireland's National Clinical Programmme recently produced clinical guidelines on pharmacotherapy for COPD. However, there is no coherent picture of the prevalence of COPD in Ireland, the characteristics of who is receiving medication for COPD and how prescribing aligns with best practice. In view of this, we evaluated medication dispensing data to estimate the age and sex specific prevalence of symptomatic COPD in Ireland, and to identify patterns of medication use which can be used to inform interventions to improve the management of this condition.

Methods We used the Primary Care Reimbursement Service database, a national repository of anonymised pharmacy claims for more than $40 \%$ of the Irish population who qualify for the General Medical Services (GMS) scheme; a public health insurance programme for those of lower income and those $\geq 70$ years. We used a cohort study design with data from 2016 , limiting our population to those aged $\geq 45$ years to help remove dispensing for asthma. We examined the distribution of all respiratory medications dispensed, and then the patterns of medication use in those likely to have COPD.

Results From the GMS eligible population (aged $\geq 45$ years) with coverage for the entire year $(n=730,832)$, there were 170,950 patients dispensed at least one respiratory medication in 2016; equating to approximately $23 \%$ of the GMS population $\geq 45$ years receiving at least one respiratory medication (21.5\% of males and $25.0 \%$ of females).

The prevalence of medication use suggestive of COPD in those aged $\geq 45$ years was $15.1 \%$ (m) and $16.2 \%$ (f). Prevalence was higher in females than males aged between 45 and
64 years $(13.1 \%$ vs. $10.1 \%, \mathrm{p}<0.0001)$; there was no gender difference in those aged 65-74 years; and prevalence was higher in males aged $\geq 75$ years $(21.7 \%$ vs. $18.9 \%$, $\mathrm{p}<0.0001)$. Prevalence peaked in both sexes in the oldest age category: $21.7 \%$ (m) and $18.9 \%$ (f). There was significant inhaled corticosteroid (ICS) use, along with underuse of LAMA therapy.

Conclusion The prevalence of medication use consistent with the management of symptomatic COPD mirrors international estimates on prevalence of COPD, and in the absence of a population-based prevalence study, can be used to inform decision making. The high use of ICS, and the under use of LAMA therapy and poor adherence of those newly initiated is of concern. We recommend the development of an educational intervention for health professionals to assist in the implementation of new national prescribing guidelines for the management of COPD.

\section{P24 ONLINE DATING AND SEXUALLY TRANSMITTED INFECTIONS IN ENGLAND: AN ECOLOGICAL STUDY USING GOOGLE TRENDS DATA}

$1,{ }^{2}$ LKRP Jennings* ${ }^{2} \mathrm{C}$ Kypridemos. ${ }^{1}$ School of Public Health, Health Education England
North West, Greater Manchester, UK; ${ }^{2}$ Department of Public Health and Policy, University of
Liverpool, Liverpool, UK

10.1136/jech-2019-SSMabstracts. 175

Background Online dating, and the use of dating smartphone applications has been theorised as a driver behind recent increases in some sexually transmitted infections (STIs). Existing literature is mixed, but some studies demonstrate an increase in high-risk sexual behaviours or STIs in online daters. However, many previous studies have a narrow study population and often rely on survey or interview data, which may not be representative. This ecological study aimed to investigate the association between online dating and STIs by using existing aggregated population-level data.

Methods We used official Public Health England STI incidence data by county or unitary authority in England for 2017. Based on Google Trends internet search data for the names of frequently used dating applications, we constructed a proxy measure for dating application usage, by English location in 2017. We named this measure 'proxy dating app score'. We used generalised linear modelling to explore the association between STI incidence and ranked proxy dating app score while controlling for known confounders (area index of multiple deprivation, proportion of people of black ethnicity in the population, and proportion of people aged under 25 in the population), assuming multiplicative effects. We performed extensive sensitivity analysis to test our assumptions, including a binomial regression model with STI incidence rates as the dependent variable. We used SPSS v22 to conduct the analysis.

Results In our main analysis, we observed a positive association between STI incidence and ranked proxy dating app score. Every increase in proxy dating app score rank was linked to a $7 \%(95 \% \mathrm{CI}: 4 \%$ to $11 \%, \mathrm{p}<0.01)$ rise in the count of STIs. Area deprivation and proportion of people of black ethnicity were also positively associated with count of STIs, $19 \%$ (95\% CI: $13 \%$ to $25 \%$ ) and $23 \%$ (95\% CI: $20 \%$ to $27 \%$ ) respectively, while the proportion of people aged under 25 was not a significant predictor. The direction of the associations did not change in the sensitivity analysis. 
Conclusion Our study provides further evidence for the potential link between dating applications usage and STIs at a population level. We used an emerging data source (Google Trends data) to avoid selection and desirability biases that more traditional methodologies may have. However, ecological fallacy and the use of a proxy measure for online dating applications usage are the main limitations of our approach. A well-designed longitudinal study is justified and necessary to provide robust, actionable evidence for public health priorities and strategy.

\section{P25 A DESCRIPTIVE CROSS SECTIONAL STUDY OF GPS KNOWLEDGE OF AND ATTITUDES TOWARDS THE MANAGEMENT OF BEHAVIOURAL AND PSYCHOLOGICAL SYMPTOMS OF DEMENTIA}

AA Jennings*, SBF Dhuny. Dept. of General Practice, University College Cork, Cork, Ireland

\subsection{6/jech-2019-SSMabstracts. 176}

Background Most people living with dementia will experience behavioural and psychological symptoms of dementia (BPSD) such as aggression or agitation at some point in their illness. However, evidence suggests that the current management of BPSD in primary care is sub-optimal. Non-pharmacological strategies are recommended first-line in BPSD but uptake of these strategies is low. Despite their adverse effects and minimal effectiveness antipsychotics are frequently employed to manage BPSD. The aim of this study was to explore general practitioners (GPs) knowledge of and attitudes towards the management of BPSD with a view to informing future interventions.

Methods This was a descriptive cross-sectional study. An anonymous postal questionnaire was sent in May 2018 to a census sample of all GPs in two counties in southern region of Ireland; county Kerry and county Cork. The questionnaire was adapted from a previous study and piloted with three GPs. Based on the sample size calculations, 209 responses were required to adequately power the study. All responses were coded and SPPS v25 was used for statistical analysis.

Results Of the 456 questionnaires sent 168 completed questionnaires were returned representing a response rate of $36.8 \%$. The sample was representative of GPs nationally. $62.5 \%(105 / 168)$ of respondents had a nursing home commitment. GPs unanimously believed that antipsychotics, benzodiazepines and antidepressants did not benefit all patients with BPSD. The majority of GPs (60.7\%) agreed they required more training and experience to improve their management of BPSD. 'Lack of resources in the primary care team' was cited as the main barrier to GPs recommending non-pharmacological management for BPSD. However, 69\% of GPs reported they routinely recommended non-pharmacological interventions before medication to manage BPSD. Nursing staff were identified as the group of people that most influenced the GPs prescribing of antipsychotic medications. The majority of respondents $(52.4 \%, 84 / 168)$ said they did not have a repeat prescribing policy for patients with dementia on antipsychotics. $63.1 \%(106 / 168)$ of GPs were concerned that withdrawing medication would impact negatively on the quality of life of the person with dementia leading to a return of BPSD. No association was found between years of experience in primary care and confidence to withdraw medication in BPSD $(\mathrm{p}=0.25)$.

Conclusion This study identified several factors influencing the prescription of antipsychotics in BPSD as well as barriers to recommending non-pharmacological strategies. These findings can be used to guide future interventions aimed at improving the management of BPSD in primary care.

\section{P26 EXPLORING THE ACCEPTABILITY AND FEASIBILITY OF USING ACTIVITY MONITORS TO SUPPORT INCREASED PHYSICAL ACTIVITY WITHIN AN EXERCISE REFERRAL SCHEME FOR ADULTS WITH, OR AT RISK OF, A CHRONIC HEALTH CONDITION}

${ }^{1} \mathrm{MJ}$ Kelson*, ${ }^{2} \mathrm{~J}$ Hawkins, ${ }^{3} \mathrm{M}$ Edwards, ${ }^{4} \mathrm{~L} \mathrm{McC}$ Connon, ${ }^{4} \mathrm{~B}$ Hallingberg, ${ }^{5} \mathrm{E}$ Oliver, ${ }^{6} \mathrm{~J}$ Charles, ${ }^{6} \mathrm{R}$ Tudor Edwards, ${ }^{4} \mathrm{~S}$ Murphy, ${ }^{7} \mathrm{~S}$ Simpson. ${ }^{1}$ Department of Statistical Science, University of Exeter, Exeter, UK; ${ }^{2}$ School of Social Sciences, Cardiff University, Cardiff, UK; ${ }^{3}$ School of Medicine, Cardiff University, Cardiff, UK; ${ }^{4}$ Decipher, School of Social Sciences, Cardiff University, Cardiff, UK; ${ }^{5}$ Department of Sport and Exercise Sciences, Durham University, Durham, UK; ${ }^{6}$ Centre for Health Economics and Medicines Evaluation, Bangor University, Bangor, UK; ${ }^{7}$ MRC/CSO Social and Public Health Sciences Unit, University of Glasgow, Glasgow, UK

\subsection{6/jech-2019-SSMabstracts. 177}

Background Whilst there is evidence for initial effectiveness of exercise referral schemes for increasing physical activity, evidence of long-term effects is limited. In Wales, a trial of the National Exercise Referral Scheme [NERS] showed small but significant impacts on physical activity at 12-month follow-up. Technologies such as activity monitors may enhance long-term maintenance of activity by facilitating goal setting and progress monitoring and supporting intrinsic motivation. This pilot trial explored the acceptability and feasibility of implementing accelerometry-based activity monitors within NERS.

Methods New NERS participants (mean age $=57,65 \%$ female) were randomised to receive either an activity monitor alongside NERS $(n=88)$ or usual practice $(n=68)$. Participants completed questionnaires at baseline, 16-weeks and 52-weeks. Twenty intervention participants and 12 NERS staff completed interviews at 4-weeks and 52-weeks.

Results Findings suggest that participant experiences of utilising the activity monitors were mixed. Approximately half of participants reported that the devices were easy to use $(49 \%)$ and met their expectations (57\%). In interviews, some participants reported that the monitors helped them to become more aware of their physical activity levels and increased their motivation. Barriers to acceptability included general and device-specific wearability and technological problems, such as device malfunctioning and computer compatibility issues. Staff also reported device- and context specific technological barriers to implementing the monitors alongside usual practice. Conclusion Whilst some findings were device-specific, there are broader lessons for future research and practice incorporating activity monitoring devices into physical activity interventions such as implications for delivery staff time and training. 